\title{
Geometry Optimization Approaches of Inductively Coupled Printed Spiral Coils for Remote Powering of Implantable Biomedical Sensors
}

\author{
Sondos Mehri, ${ }^{1,2}$ Ahmed Chiheb Ammari, ${ }^{3,4}$ \\ Jaleleddine Ben Hadj Slama, ${ }^{2}$ and Hatem Rmili ${ }^{3}$ \\ ${ }^{1}$ ENIT, University of Tunis El Manar, Belvedere, 1002 Tunis, Tunisia \\ ${ }^{2}$ SAGE, National Engineering School of Sousse, ENISo, University of Sousse, 4054 Sousse, Tunisia \\ ${ }^{3}$ Renewable Energy Research Group, Department of Electrical \& Computer Engineering, Faculty of Engineering, \\ King Abdulaziz University, Jeddah 21589, Saudi Arabia \\ ${ }^{4}$ MMA Laboratory, National Institute of the Applied Sciences and Technology, Carthage University, 1080 Tunis, Tunisia
}

Correspondence should be addressed to Ahmed Chiheb Ammari; ac.ammari@yahoo.fr

Received 27 November 2015; Revised 22 February 2016; Accepted 6 March 2016

Academic Editor: Alberto J. Palma

Copyright (C) 2016 Sondos Mehri et al. This is an open access article distributed under the Creative Commons Attribution License, which permits unrestricted use, distribution, and reproduction in any medium, provided the original work is properly cited.

\begin{abstract}
Electronic biomedical implantable sensors need power to perform. Among the main reported approaches, inductive link is the most commonly used method for remote powering of such devices. Power efficiency is the most important characteristic to be considered when designing inductive links to transfer energy to implantable biomedical sensors. The maximum power efficiency is obtained for maximum coupling and quality factors of the coils and is generally limited as the coupling between the inductors is usually very small. This paper is dealing with geometry optimization of inductively coupled printed spiral coils for powering a given implantable sensor system. For this aim, Iterative Procedure (IP) and Genetic Algorithm (GA) analytic based optimization approaches are proposed. Both of these approaches implement simple mathematical models that approximate the coil parameters and the link efficiency values. Using numerical simulations based on Finite Element Method (FEM) and with experimental validation, the proposed analytic approaches are shown to have improved accurate performance results in comparison with the obtained performance of a reference design case. The analytical GA and IP optimization methods are also compared to a purely Finite Element Method based on numerical optimization approach (GA-FEM). Numerical and experimental validations confirmed the accuracy and the effectiveness of the analytical optimization approaches to design the optimal coil geometries for the best values of efficiency.
\end{abstract}

\section{Introduction}

The treatment of a growing number of ailments has become real thanks to the revolution in semiconductor technology and the steady growth of integrated circuit (IC) design for implantable biomedical systems. An implantable electronic biomedical sensor device is generally composed of an external controller and an implantable unit [1]. The external controller sends command to the implantable unit and processes received data from the implants through an interface unit which needs powering to perform its role. Among the main reported approaches, inductive links are the most commonly used methods for remote powering of such devices. This method does not require any transcutaneous wires, which may cause infections, or any implantable batteries which need to be replaced at the end of their lifetime. The power efficiency is the most important characteristic to be considered when designing inductive links to transfer energy to implantable sensing devices [2]. Maximum power efficiency is obtained for maximum coupling factors of the coils. This parameter is generally limited since the coupling between inductors is usually very small. Improving power efficiency of an inductive link is crucial and essential to minimize the size of the external energy source, the electromagnetic heating of the tissue, the interference with other devices, and imperatively the safety of the patient. Therefore, attention must be paid 
to the link design in order to obtain high transferred power efficiency [2]. This includes proper optimization of coil geometries [3], appropriate design of primary coil drivers [4], procedures to operate the link as close as possible to its theoretical efficiency limit [5], and also servo loops that may be used to automatically adjust the link parameters to changing coupling and load conditions [6].

We are particularly concerned with the geometric optimization of inductively coupled printed spiral coils (PSC) for powering biomedical implantable sensor devices. This optimization is a laboured assignment given the large number of parameters characterizing the link performance. The objective is to optimize, within an acceptable design time, the PSC geometric parameters such that the maximum link efficiency is guaranteed. This optimization problem is of high dimensions, has high degree of nonlinearity, and is thus hard to solve. In this context, two main classes of optimization methods are generally used: analytic and numeric approaches. The analytic approaches are based on simple mathematical models that approximate both coil parameters and the link power efficiency. They are implemented using either iterative design procedures or advanced global search metaheuristics. The Iterative Procedure is a traditional popular approach for optimizing the link coil geometries $[7,8]$. Advanced population based search strategies implementing Genetic Algorithms [9, 10] or particle swarm optimizers [11] can also be used for inductive link optimizations. Such global approaches aim to efficiently explore the search space such that near-optimal solutions are obtained and rapid convergence is ensured [12]. For the numeric approaches, the optimization is also implemented through conventional global search techniques such as Genetic Algorithms [13] or particle swarm optimizers [14]. The difference is that, in this case, the objective function is evaluated by numerical simulation using specific finite element software packages. Numeric optimization methods have been previously implemented for many RFID [15] and antenna [16] design cases.

This work aims to implement appropriate optimization approaches capable of achieving optimal link designs within acceptable design times. Analytical approaches implementing either Iterative Procedures (IP) or Genetic Algorithms (GA) are first proposed. These approaches were originally presented in [17]. This paper starts by reviewing the IP approach and details the implementation of its different steps. The GA optimization for the chosen implant is then developed and appropriate selection, crossover, and mutation operators are carefully designed to ensure rapid convergence of the algorithm. Moreover, a purely numerical simulation based optimization, GA-FEM, is developed. GAFEM consists in coupling finite element numeric simulation method (FEM) with GA based search strategy $[16,18]$. The outperformance of GA-FEM is demonstrated. Comparatively, efficiency degradations of proposed IP and GA designs are evaluated. Comparisons with a previous design studied for the same implant in [19] are also performed. At the end, experimental measurements are implemented to validate the different design approaches. Reasonable errors are observed for the analytical results despite the approximate

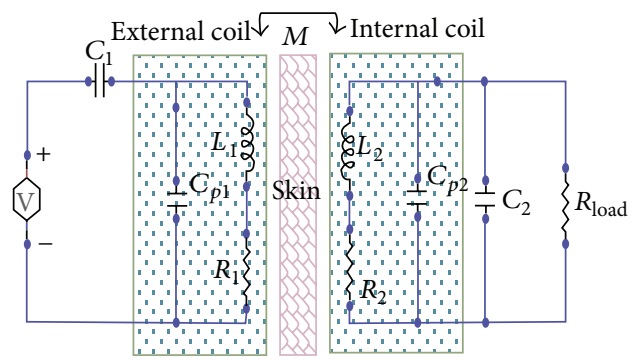

FIgURE 1: Simplified model of the inductive power link.

mathematical models being used. Comparing IP to GA, the accuracy and the effectiveness of GA are confirmed.

The remainder of the paper is as follows. The next section explains the basic theoretical background of inductive link modeling and efficiency calculations. For an inductive link optimal geometric design, the Iterative Procedure (IP) together with the GA based analytical optimization approaches is presented in Section 3. The GA-FEM numeric optimization approach is described in Section 4. Section 5 presents the experimental validation with a comparative analysis between the different design approaches. Conclusions are given in the last section.

\section{Theoretical Background}

This section first introduces the circuit model of an inductive link, and then simple mathematical models that approximate coil parameters and transfer power efficiency are presented. A basic assumption that the secondary coil of the link is embedded in the human body is ignored since, referring to [20-22], it is shown that losses through biological tissues and liquids are negligible at frequencies between several hundred $\mathrm{kHz}$ to $20 \mathrm{MHz}$. For the study, $13.56 \mathrm{MHz}$ operating frequency is selected. Thus, the surrounding media of the coils are neglected for the following coil modeling, simulation, and experimentation.

2.1. Inductive Link Modeling. The behavior of an inductive link comprising a primary circuit and a secondary circuit can be treated at two different levels of abstraction: a description of electromagnetic fields and a description of the circuit level. The latter description can be used to deduct the characteristics of the link. However, key circuit component such as inductors, parasitic elements, and magnetic coupling coefficients can be extracted using only the electromagnetic field description. Figure 1 shows a simplified diagram of an inductive power link. $L_{1}$ and $L_{2}$ are, respectively, the primary and secondary coils. The primary coil is fixed outside the body, whereas the secondary coil is attached to electronics of the implant that are embedded in the human body. Coil windings are characterized by their respective parasitic resistance $\left(R_{1}, R_{2}\right)$ and capacitance $\left(C_{p 1}, C_{p 2}\right)$.

To increase the transfer efficiency, both sides of the link are tuned to the same resonant frequency. This can be implemented by adding two capacitors $C_{1}$ and $C_{2}$, respectively, to the primary and secondary circuits to form primary and secondary LC resonant circuits. There are four doubly tuned 


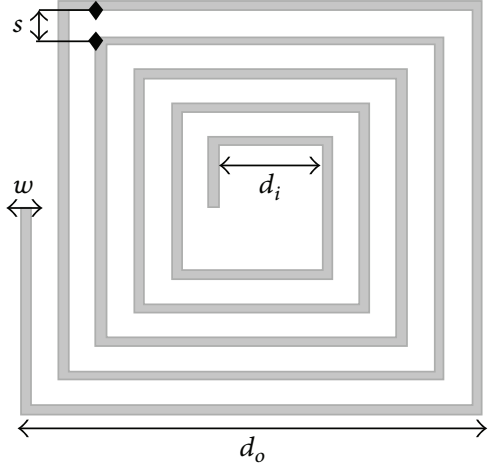

FIGURE 2: Geometry of a square spiral coil.

link choices involving all possible combinations of current and voltage input and output [4]. For example, a seriesresonant has a current-source output characteristic and a voltage-source output is achieved through parallel resonance of the secondary circuit. In Figure 1, the primary circuit uses a serial LC circuit to provide low impedance to the driving side. The secondary circuit is tuned in parallel with better drive nonlinear rectifier loads. A parallel-tuned setup has the additional advantage that the parasitic coil capacitor is absorbed in the resonance capacitor $C_{2}$, allowing for higher transfer frequencies and thus smaller coils [4].

\subsection{Planner Spiral Coil Quality Factor and Mutual Inductance.} Applying a magnetic field at the primary coil will induce a current flowing in the secondary circuit. The value of the induced current is related to $L_{1}$ and $L_{2}$ inductances of both primary and secondary coils. For the envisaged applications, a square spiral coil form is selected given that, for a defined boundary box, square spiral coils enable the maximum power transfer area [8]. For the chosen square spiral planar coil (Figure 2), the inductance is calculated according to the following formula [19]:

$$
L=\frac{1.27 \cdot \mu_{0} \cdot n^{2} d_{\mathrm{avg}}}{2}\left[\ln \left(\frac{2.07}{\varphi}\right)+0.18 \varphi+0.13 \varphi^{2}\right],
$$

where $n$ is number of turns

$$
\begin{aligned}
d_{\mathrm{avg}} & =\frac{\left(d_{i}+d_{o}\right)}{2} ; \\
\varphi & =\frac{\left(d_{o}-d_{i}\right)}{\left(d_{o}+d_{i}\right)}
\end{aligned}
$$

with $d_{o}$ and $d_{i}$ being the outer and inner diameter, respectively.

(i) Quality Factor. The inductor quality factor is an important parameter that affects the link power efficiency. It is related to the parasitic resistance and capacitance of the inductor. Taking into account the skin effect, the total parasitic resistance can be calculated as follows [8]:

$$
R_{s}=R_{\mathrm{dc}} \frac{t_{c}}{\delta \cdot\left(1-e^{-t_{c} / \delta}\right)}
$$

with $\delta$ being the skin metal depth and $R_{\mathrm{dc}}$ the DC resistance, expressed as follows:

$$
\begin{aligned}
R_{\mathrm{dc}} & =\rho_{c} \frac{l_{c}}{w \cdot t_{c}} ; \\
\delta & =\sqrt{\frac{\rho_{c}}{\pi \cdot \mu \cdot f}} ; \quad \mu=\mu_{r} \cdot \mu_{0},
\end{aligned}
$$

where $l_{c}$ is total length of the conductor, $t_{c}$ is conductor thickness, $w$ is conductor width, $\rho_{c}$ is metal resistivity, $\mu$ is permeability constant, and $\mu_{r}$ is relative permeability of the conductor.

Ignoring the parasitic capacitance of the circuit, the quality factor of the Printed Circuit Coil (PSC) can be expressed as $Q=\omega L / R_{s}[8]$.

(ii) Coils Mutual Inductance. The mutual inductance is a key parameter for inductive power transfer. Assuming perfect alignment, the total mutual inductance between the two coils is expressed by

$$
M=g \sum_{i=1}^{n_{1}} \sum_{j=1}^{n_{2}}\left(M_{i j}, r_{i}, r_{j}, d_{12}\right),
$$

where $g$ is factor depending on the shape of the coil ( $g=1.3$ for square shape), $n_{1}$ is number of turns of the primary coil, $n_{2}$ is number of turns of the secondary coil, $r_{i}$ is radius of $i$ th turn in primary coil, $r_{j}$ is radius of $j$ th turn in secondary coil, and $d_{12}$ is distance between coils.

2.3. Inductive Link Power Efficiency. The highest voltage gain and efficiency across an inductive link (Figure 1) can be achieved when both LC tanks are tuned at the link operating frequency. Assuming $\omega=\omega_{01}=\omega_{02}$, the efficiency of the transmitted power from source to load is dominated by primary and secondary side efficiencies, $\eta_{1}$ and $\eta_{2}$, and can be expressed by

$$
\begin{aligned}
\eta_{12} & =\eta_{1} \cdot \eta_{2} \\
& =\frac{X\left(R_{2} / R_{\text {load }}\right)}{\left(1+R_{2} / R_{\text {load }}+X\left(R_{2} / R_{\text {load }}\right)\right)\left(1+R_{2} / R_{\text {load }}\right)},
\end{aligned}
$$

where

$$
X=\frac{M^{2} \cdot \omega^{2}}{R_{1} \cdot R_{2}}=k^{2} \cdot Q_{1} \cdot Q_{2}, \quad \text { with } k=\frac{M}{\sqrt{L_{1} \cdot L_{2}}},
$$

where $k$ is the coupling coefficient and $Q_{1}$ and $Q_{2}$ are the quality factors of the unloaded primary and secondary coils, respectively.

By taking $R_{\text {load }}$ as optimum, the maximum efficiency can be calculated as follows:

$$
\eta_{12}=\frac{k^{2} Q_{1} Q_{2}}{\left(1+\sqrt{1+k^{2} Q_{1} Q_{2}}\right)^{2}} .
$$

For more details about how these formulas are obtained, one can consult $[19,21,22]$. 


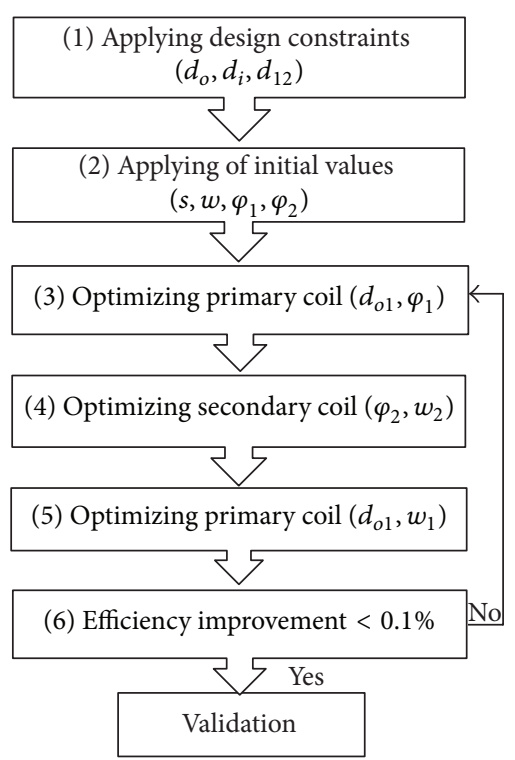

FIGURE 3: Iterative design procedure flowchart.

\section{Analytic Design IP and GA Based Approaches}

This section presents Iterative Procedure (IP) and Genetic Algorithm (GA) analytical approaches to optimally design the coil geometries of two inductively coupled printed spiral coils for powering a particular biomedical implantable system. The chosen biomedical implantable sensor is used for a laboratory mouse and aims for biomedical and genetic researches to investigate new treatments as presented in [19]. For such design approaches, some parameters are application constrained and others have to be carefully chosen to guarantee the best efficiency values of the link.

3.1. The Iterative Procedure (IP). The implemented Iterative Procedure consists of six steps as presented in the flowchart of Figure 3. follows.

Each step in this figure is explained and detailed as

(1) Applying Design Constraints. There is a set of constrained parameters that are imposed by factors associated with the chosen implant system. These parameters usually define the size constraints as limited by where the implant is located. Other factors are related to the fabrication technology. This consists in the minimum size features that result in acceptable manufacturing. For our case, the constrained parameters and their values are listed in Table 1.

(2) Initial Values. For this study, the primary coil outer diameter $d_{o 1}$, the spacing between the conductors $\left(s_{1}, s_{2}\right)$, and the width of conductors $\left(w_{1}, w_{2}\right)$ for the primary and secondary coils are the selected variables to be optimized. A set of initial values for these variables is defined before
TABLE 1: Design parameters limited by implant and fabrication technology.

\begin{tabular}{lc}
\hline Parameters & Value \\
\hline Secondary coil outer diameter $\left(d_{o 2}\right)$ & $20(\mathrm{~mm})$ \\
Distance between coils $\left(d_{12}\right)$ & $30(\mathrm{~mm})$ \\
Minimum coils inner diameters $\left(d_{i 1}\right.$ and $\left.d_{i 2}\right)$ & $5(\mathrm{~mm})$ \\
Minimum width of conductor ${ }^{*}(w)$ & $150(\mu \mathrm{m})$ \\
Minimum spacing between conductors* ${ }^{*}(s)$ & $150(\mu \mathrm{m})$ \\
Operating frequency $(f)$ & $13.56(\mathrm{MHz})$ \\
Substrate thickness $\left(t_{s}\right)$ & $1.5(\mathrm{~mm})$ \\
Conductor thickness $\left(t_{c}\right)$ & $38(\mu \mathrm{m})$ \\
${ }^{*}$ The conductor is copper on FR4 printed circuit board. &
\end{tabular}

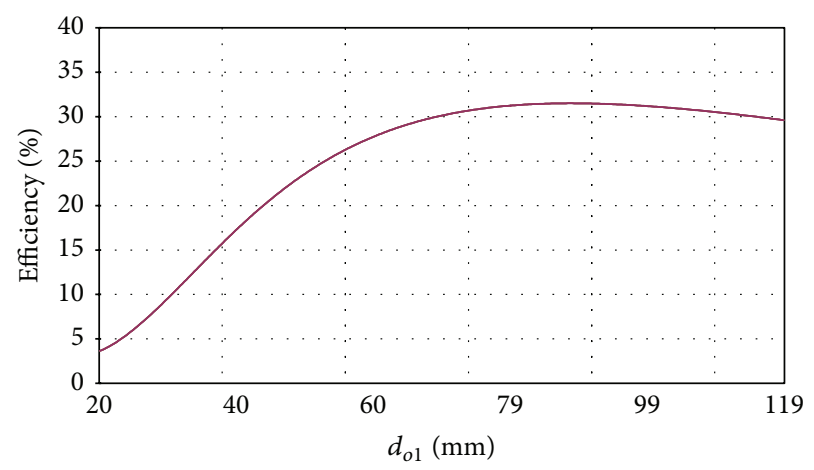

Figure 4: Calculated efficiency $\left(\eta_{12}\right)$ versus $\left(d_{o 1}\right)$ for Step 3 optimization.

starting the iterative optimization process. As shown in [8], a good choice for $d_{o 1}$ initial value would be

$$
d_{o 1}=d_{12} * \sqrt{2}=42 \mathrm{~mm}
$$

The initial values selected for the other parameters are set to their minimum values as follows: $s_{1}=s_{2}=0.15 \mathrm{~mm}$ and $w_{1}=w_{2}=0.15 \mathrm{~mm}$.

(3) Optimizing the Size and Fill Factor of Primary PSC $\left(d_{o 1}, \varphi_{1}\right)$. The third step of the Iterative Procedure aims to optimize the primary side efficiency. Plugging the coil parameters initial values defined in previous steps into (1) to (8), the link efficiency $\eta_{12}$ is calculated for different $d_{o 1}$ and $\varphi_{1}\left(\varphi_{1}=\left(d_{o 1}-d_{i 1}\right) /\left(d_{o 1}+d_{i 1}\right)\right)$ varying in a wide range around their initial values. Since $s_{1}$ and $w_{1}$ are kept constant for this step, modifying $d_{o 1}$ and $\varphi_{1}$ directly affects $k, Q_{1}$, and $\eta_{12}$ as given by (8). It is obtained that $\eta_{12}$ efficiency results, calculated for different $d_{o 1}$ and $\varphi_{1}$, converge to the highest values for $\varphi_{1}>0.62$. Selecting $\varphi_{1}=0.62, \eta_{12}$ is computed versus $d_{o 1}$ as reported in Figure 4 .

Figure 4 clearly shows that the maximum efficiency values are obtained for $d_{o 1}$ within 80 to $100 \mathrm{~mm}$ range. For minimal primary coil sizes, the best choice for $d_{o 1}$ is set to $80 \mathrm{~mm}$. Setting $\varphi_{1}=0.62$, the inner diameter for the primary coil, $d_{i 1}$, is calculated as $d_{i 1}=10 \mathrm{~mm}$. For this step, almost $32 \%$ of total efficiency is obtained. This efficiency value should improve once $w_{1}$ and the secondary coils are optimized as shown next. 


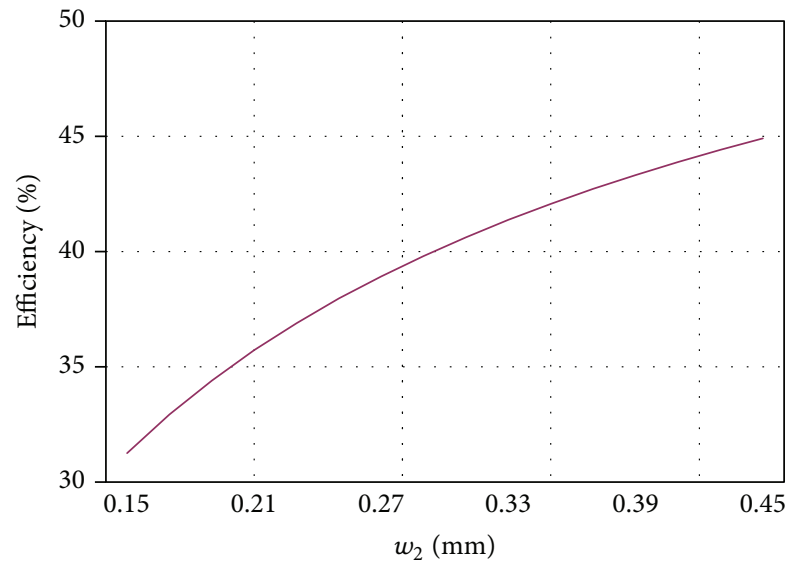

Figure 5: Calculated efficiency $\left(\eta_{12}\right)$ versus $\left(w_{2}\right)$ for Step 4 optimization.

(4) Optimizing Fill Factor and Line Width of Secondary PSC $\left(\varphi_{2}, w_{2}\right)$. The geometry of the primary PSC was temporarily resolved in Step 3. In this step, we focus on the secondary coil. Using the parameter values issued from previous steps, the link efficiency $\eta_{12}$ is calculated while seeping $\varphi_{2}\left(\varphi_{2}=\right.$ $\left.\left(d_{o 2}-d_{i 2}\right) /\left(d_{o 2}+d_{i 2}\right)\right)$ around its initial value and $w_{2}$ set to $w_{2 \min }=0.15 \mathrm{~mm}$. It is shown that high efficiency peaks are obtained for $\varphi_{2}>0.65$ values. However, $\varphi_{2}>0.65$ leads to $d_{i 2}<4 \mathrm{~mm}$ which is less than $d_{i \min }$. We choose $d_{i 2}=11 \mathrm{~mm}$ to leave more space for an electronic chip to be placed at the center of the secondary PSC. This will reduce $\varphi_{2}$ to 0.29 . Using this $\varphi_{2}$ value, $\eta_{12}$ is reported in Figure 5 for $w_{2}$ varying from $w_{2 \min }=0.15 \mathrm{~mm}$ to $0.45 \mathrm{~mm}$. Figure 5 illustrates that the efficiency increases almost linearly with increasing $w_{2}$ values. However, it is known that proximity effect losses increase with increasing metal width [23]. To reduce proximity effect losses without compromising too much efficiency, we select $w_{2}=0.3 \mathrm{~mm}$ which is an acceptable trade-off value. This yields $41 \%$ efficiency which will again improve once $w_{1}$ is optimized.

(5) Optimizing Outer Diameter and Line Width of Primary PSC $\left(d_{o 1}, w_{1}\right)$. In this step, the primary PSC is visited back. The width of the conductor $w_{1}$ is increased from $w_{1 \min }$ to its optimal value and the outer diameter $d_{o 1}$ is increased from its original value. Increasing $w_{1}$ comes with decreasing the coil resistance $R_{\mathrm{dc}}$ as defined by (4). This leads to higher quality factor $Q_{1}$ of the primary coil and results in better $\eta_{12}$ efficiency values. However, increasing $w_{1}$ also requires larger coils with higher $d_{o 1}$ values. Observing $\eta_{12}$ curves for various $d_{o 1}$ and $w_{1}$ has shown that the highest efficiency values are guaranteed for $d_{o 1}>75 \mathrm{~mm}$. Considering $d_{o 1}=75 \mathrm{~mm}$ along with $d_{i 1}=$ $8 \mathrm{~mm}$ leads to $\varphi_{1}=0.8$. Using these settings for $d_{o 1}$ and $\varphi_{1}$, $\eta_{12}$ is calculated and reported versus increasing $w_{1}$ (Figure 6). As shown in Figure 6, $\eta_{12}$ improves with increasing $w_{1}$ and moves towards a saturation value around $w_{1}=3 \mathrm{~mm}$. To minimize the mutual coupling between conductors of the same coil [24], we select $w_{1}=2.2 \mathrm{~mm}$ as an optimal value with almost $75 \%$ efficiency achieved.

In comparison with Figure 4 (Step 3), we are achieving in this step an efficiency improvement of about $45 \%$.
TABle 2: Optimal inductive link coil designs by using Iterative Procedure.

\begin{tabular}{lcc}
\hline Parameters & $\begin{array}{c}\text { Primary } \\
\text { coil }\end{array}$ & $\begin{array}{c}\text { Secondary } \\
\text { coil }\end{array}$ \\
\hline Outer diameter $\left(d_{o}\right)$ & $80(\mathrm{~mm})$ & $20(\mathrm{~mm})$ \\
Inner diameter $\left(d_{i}\right)$ & $10(\mathrm{~mm})$ & $11(\mathrm{~mm})$ \\
Width of conductor $(w)$ & $2.5(\mathrm{~mm})$ & $0.3(\mathrm{~mm})$ \\
Spacing between conductors $(s)$ & $7.5(\mathrm{~mm})$ & $0.6(\mathrm{~mm})$ \\
Number of turns $(n)$ & 4 & 5 \\
$\eta_{12}$ at $f=13.56 \mathrm{MHz}$ & \multicolumn{2}{c}{$78 \%$} \\
\hline
\end{tabular}

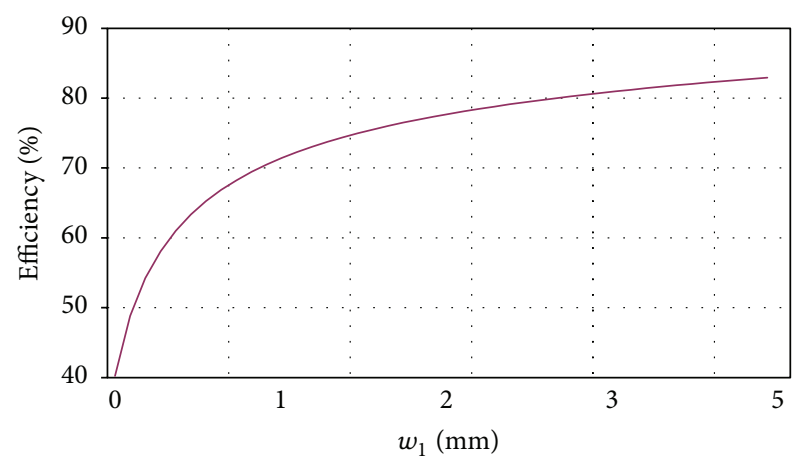

FIgURE 6: Calculated efficiency $\left(\eta_{12}\right)$ versus $\left(w_{1}\right)$ for Step 5 optimization.

(6) Iteration by Going to Step 3, Optimized Design, and Efficiency Results. Efficiency values from Step 5 significantly improve in comparison to Step 3. However, we can achieve further improvement by iterating through Steps 3-5. Iterations can continue until less than $0.1 \%$ improvement is obtained per iteration. Finally, the optimal link parameters realized using the IP approach are summarized in Table 2. For this optimized geometry, the link efficiency is enhanced to $78 \%$. However, during the implementation of the current IP procedure, we faced a difficulty in defining the best value for conductor widths $\left(w_{1}\right.$ and $\left.w_{2}\right)$ while simultaneously optimizing the space between conductors $\left(s_{1}\right.$ and $\left.s_{2}\right)$. This will be quite possible by using the Genetic Algorithms (GA) based approach that will allow the optimization of both $s$ and $\mathrm{w}$ parameters at the same time as it will be shown in the next section.

3.2. Genetic Algorithms (GA) Based Approach. Genetic Algorithms (GA) are population based search metaheuristics capable of generating solutions for problem optimizations using mechanisms inspired from the natural evolution process. GA uses a population with different individuals carrying chromosomes that represent the parameters to be optimized. Chromosomes are changed in a way similar to gene evolution, mainly through three operators: selection, crossover, and mutation. Chromosomes with better performances have more chances to survive into the next generation. Repeating these steps, the individuals in the population will become better and better, eventually 
reaching the optimum. The construction of a GA optimization for the studied implant is conducted along a workflow divided into five major parts as follows.

(1) System Specification. This consists in setting first the design constraints as enforced by the implant system. These constraints are those defined in Table 1 . In addition, we limited the size of the primary PSC with using $d_{o 1}=80 \mathrm{~mm}$ and choose $d_{i 2}=11 \mathrm{~mm}$ to leave some space for a chip to be placed at the center of the secondary PSC. After that, the fitness or objective function is defined along with the parameters to be optimized. For our case, $w_{1}, w_{2}, s_{1}$, and $s_{2}$ are selected as the optimization variables and the maximum power efficiency is our optimization target. The objective is to optimize the geometries of the coils, while the target efficiency is calculated using the electric parameters defined by (1) to (8).

(2) Initialize Population. In this step, all individuals are randomly generated to initialize the population with respecting upper and lower limits of the different coil parameters. The appropriate population size is carefully chosen as higher accuracy of GA is obtained for increasing population sizes. However, excessive increase in population sizes can significantly slow down the speed of the system to converge.

(3) Evaluate the Fitness of Each Chromosome in the Population. The fitness of all individuals in the population is calculated, so that best individuals are selected in a selection step. In our case, the fitness function accepts a vector of the geometric variables $w_{1}, w_{2}, s_{1}$, and $s_{2}$ to be optimized. These variables are encoded into a bit stream to be used just like a chromosome. Each bit stream represents an individual and is a combination of all optimized parameters. For each individual of the population, the actual design parameters are plugged into (1) to (8) to derive the link efficiency $\eta_{12}$ that is a measure of the fitness value for that individual. To avoid also unreasonable design solutions, upper and lower limits are assigned for the different candidate design solutions.

(4) Selection, Crossover, and Mutation. Appropriate selection, crossover, and mutation operators are implemented. Selection is a key operation in GA. It aims to select better individuals in a population. We used the Roulette wheel selection for this operator. It is a selection method based on proportional probability as presented in [25]. Some of the selected chromosomes are recombined and their genes exchanged through a crossover operation. The crossover creates better individuals by recombining some of the selected chromosomes. A crossover probability $P_{c}$ is used to determine whether an individual is going to do crossover with others or not [26]. In our study, the probability $P_{c}$ is randomly selected within $\left[P_{c \max }, P_{c \min }\right]$ range similarly as used in [27]. To avoid premature convergence, $P_{c \max }$ and $P_{c \min }$ are carefully selected to 0.9 and 0.8 , respectively. Finally, a mutation operation is necessary to create new individuals to get GA jump out of local optimums. This is implemented using a probability $P_{m}$ for a population to create new individuals. Low $P_{m}$ values may cause GA to fall rapidly into local optimums.
TABLE 3: Optimal inductive link coil designs by using genetic algorithms.

\begin{tabular}{lcc}
\hline Parameters & $\begin{array}{c}\text { Primary } \\
\text { coil }\end{array}$ & $\begin{array}{c}\text { Secondary } \\
\text { coil }\end{array}$ \\
\hline Outer diameter $\left(d_{o}\right)$ & $80(\mathrm{~mm})$ & $20(\mathrm{~mm})$ \\
Inner diameter $\left(d_{i}\right)$ & $10(\mathrm{~mm})$ & $11(\mathrm{~mm})$ \\
Conductors' width $(w)$ & $1.5(\mathrm{~mm})$ & $0.25(\mathrm{~mm})$ \\
Spacing between conductors $(s)$ & $3(\mathrm{~mm})$ & $0.35(\mathrm{~mm})$ \\
Number of turns $(n)$ & 8 & 8 \\
$\eta_{12}$ at $f=13.56 \mathrm{MHz}$ & \multicolumn{2}{c}{$80.6 \%$} \\
\hline
\end{tabular}

However, excessive $P_{m}$ values will slow down the converging speed of the method. 0.001-0.01 is a commonly used range for $P_{m}$ as presented in $[28,29]$.

(5) Meet Convergence Stopping Criteria. The algorithm ends either when a maximum number of generations are produced or when a satisfactory optimization level is reached. When the stopping criteria are met, the GA process is completed and the optimal efficiency value is extracted. Otherwise, a new population is generated as defined by the GA rules and Step 3 is restarted.

For the current design case, a MATLAB software tool implementing Genetic Algorithms is used. To run this tool, an M-file that computes the objective function to be optimized is first developed. Carrying out the GA optimization has achieved the optimal geometric parameters for the actual implant coils. The obtained optimal parameters are summarized in Table 3. Using these geometries in (1) to (8), $80.6 \%$ efficiency is obtained. This is a slightly better efficiency value in comparison with what we get using the IP approach. However, if we consider the implementation burden of IP, the GA based algorithms help perform the optimization in a more systematic and efficient way. In addition, GA took very few cycles to converge and once properly configured, it takes very little time to deliver an adequate design.

\section{GA-FEM Numeric Optimization Approach}

This section discusses a purely numeric GA-FEM optimization approach. This approach is implemented using a particular software module that is integrated within HFSS FEM simulator [18]. It consists in coupling finite element numeric simulation method with GA based search strategy. Similar techniques have been previously proposed to optimize antennas and waveguide systems [30-32]. In these previous studies, FEM simulators are connected with MATLAB to solve the related problems. For the present case, Genetic Algorithm optimization is already integrated with HFSS FEM simulator. Implementing GA-FEM consists in three main phases. For each phase, particular modeling and design tasks are required. First, initial geometry parameters with specific design constraints are inserted to restrict the search region and prevent the optimizer from creating physically meaningless designs (such as overlapping and overtaking of the geometry). The second phase runs the "GA-FEM-optimizer" that 


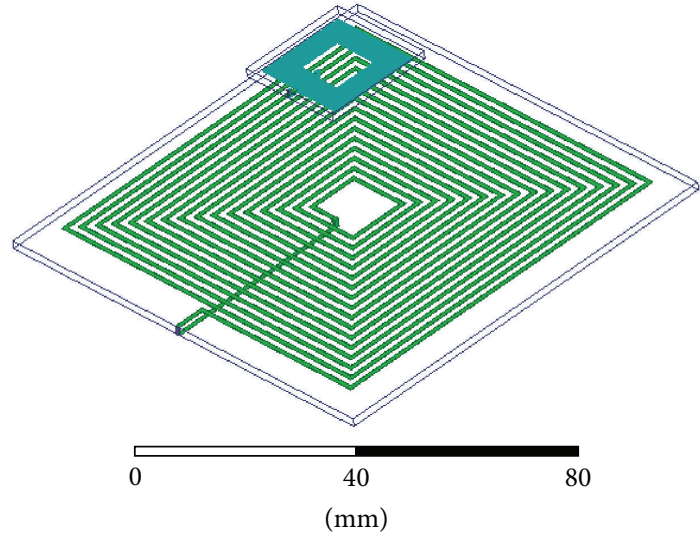

Figure 7: 3D model of initial geometry defined for GA-FEM optimization.

TABle 4: Optimal inductive link coil designs by using GA-FEMoptimizer.

\begin{tabular}{lcc}
\hline Parameters & $\begin{array}{c}\text { Primary } \\
\text { coil }\end{array}$ & $\begin{array}{c}\text { Secondary } \\
\text { coil }\end{array}$ \\
\hline Outer diameter $\left(d_{o}\right)$ & $80(\mathrm{~mm})$ & $20(\mathrm{~mm})$ \\
Inner diameter $\left(d_{i}\right)$ & $10(\mathrm{~mm})$ & $11(\mathrm{~mm})$ \\
Conductors' width $(w)$ & $1.9(\mathrm{~mm})$ & $0.7(\mathrm{~mm})$ \\
Spacing between conductors $(s)$ & $2.5(\mathrm{~mm})$ & $0.52(\mathrm{~mm})$ \\
Number of turns $(n)$ & 8 & 4 \\
$\eta_{12}$ at $f=13.56 \mathrm{MHz}$ & \multicolumn{2}{c}{$78 \%$} \\
\hline
\end{tabular}

implements a Genetic Algorithm based search to produce a new structure starting from the initial geometry settings. In this phase, a fitness or objective value function is computed and appropriate selection, crossover, and mutation operators are used.

For the current design case, the initial geometry structures for the primary and secondary coils are HFSS modeled as shown in Figure 7. The variables selected to be optimized are $w_{1}, w_{2}, s_{1}$, and $s_{2}$ and their lower and upper limits are fixed. The maximum power efficiency is again our optimization target and the fitness or objective function is defined accordingly. GA-FEM also optimizes the geometries of the coils. However, in this case, the electric parameters such as resistances, inductances, coupling constants, and quality factors of the coils are obtained using numerical simulations. The simulated electric parameters are used again with (8) to evaluate the maximum efficiency and measure the fitness values of all individuals for a given generation. To move from one generation to the next, the same settings we used for GA are here implemented for selection, crossover, and mutation operators with the same rate of mutation and crossover recombination. 90\% target efficiency objective is fixed and 60 hours maximum simulation time is defined for the system to converge to the desired goal. The simulation ends either if the desired optimization level is met or if the maximum simulation time is reached.

Running GA-FEM numeric optimization, we were not able to achieve the $90 \%$ desired target. However, we ended

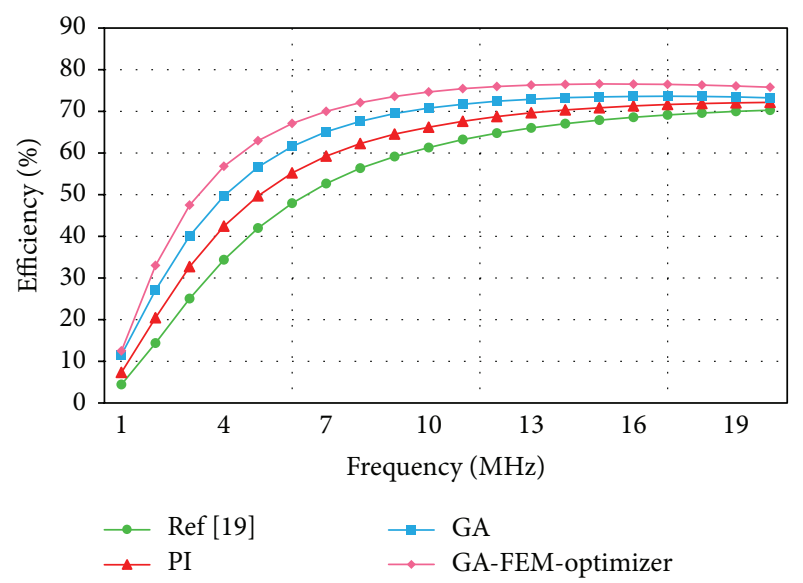

FIgure 8: Simulated efficiency values for GA-FEM, GA, and IP design optimizations compared to [19].

after 60 hours of simulation time with the optimized geometries which is illustrated in Table 4

The simulated efficiency values of the optimized coils with GA-FEM are given in Figure 8 together with the efficiency results for the PI, GA, and [19] optimized designs. These efficiency values are reported versus frequency as they are based on the coils electric parameters that are simulated for $[0-20 \mathrm{MHz}]$ frequency range. Particularly, for $13.56 \mathrm{MHz}$ operating frequency, GA-FEM is capable of achieving $78 \%$ efficiency as reported in Table 4. In reference to GA-FEM, the efficiency losses of the approximate analytic approaches are evaluated. As reported in Figure 8, 9.7\% and 5.7\% efficiency degradation are obtained at the operating frequency for IP and GA, respectively. However, more than $16 \%$ efficiency losses are observed when comparing GA-FEM to [19]. This outlines the outperformance of the proposed analytical IP and GA methods in comparison with [19].

Compared to IP and GA analytical approaches, the outperformance of GA-FEM is clearly illustrated in Figure 8. However, if we consider the long simulation time of GAFEM and also the modeling and design efforts required to implement it, one can easily conclude that GA, although it is relying on approximate analytic circuit modeling, is capable of competing with rapid and systematic design optimizations and with comparable performances. In addition, the GA optimized design can also serve to define the initial design structure for GA-FEM optimization to get further performance improvement with augmented convergence speed. Actually, when GA is used to provide the initial parameters for GA-FEM (GA-FEM-initialized), the same efficiency of the previous GA-FEM optimal design (78\%) is obtained after only $3 \mathrm{~h}: 52 \mathrm{mn}$ of computing time in comparison with 60 hours previously needed. However, if we leave the simulations complete to the end, the system will converge to a design with even better efficiency values as shown in Figure 9. In this figure, around $81 \%$ efficiency is observed at $13.56 \mathrm{MHz}$ operating frequency for GA-FEM-initialized with GA. Actually, GAFEM-initialized achieves an improved performance in comparison with GA-FEM. Moreover, noticeable convergence 


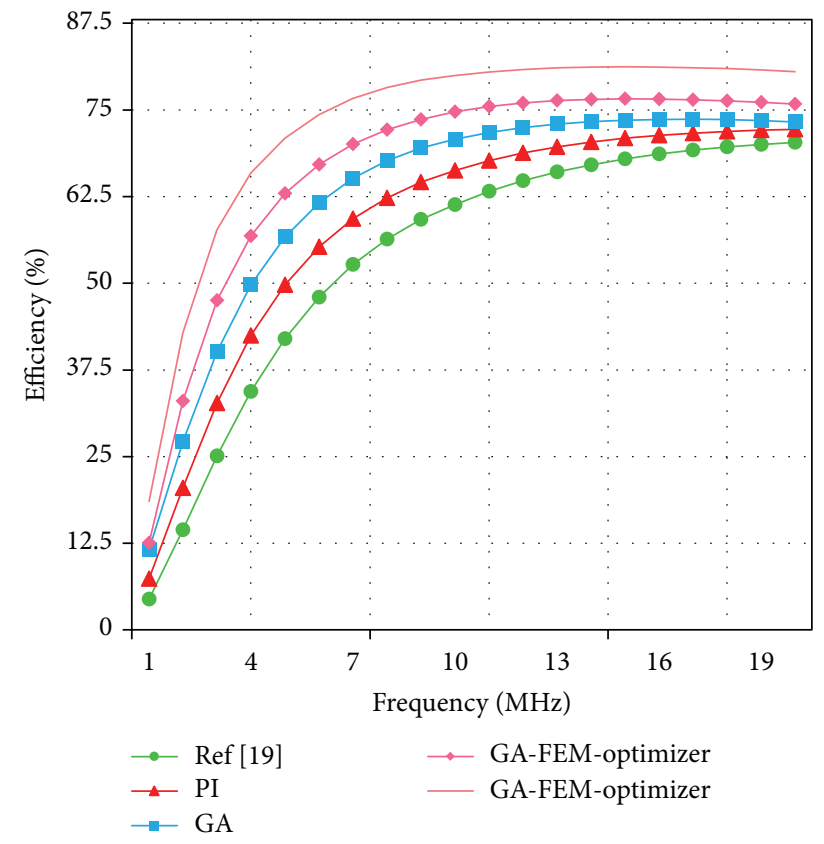

FIGURE 9: Simulated efficiency values for GA-FEM-initialized in comparison with GA-FEM, GA, IP, and [19].

speed acceleration is observed which represents an additional outcome for the proposed GA analytical based optimization.

\section{Experimental Measurement Results}

To confirm the validity of the proposed approaches, experimental measurements are required. For this aim, the different designed coils are fabricated on printed circuit boards (PCB) using $0.035 \mathrm{~mm}$ copper and FR4 Epoxy substrates $[8,22]$. The first fabricated PCB is related to the primary and secondary coils of reference design [19]. The second and third designed coils are fabricated according to the optimal geometries designed, respectively, with IP and GA approaches. The last $\mathrm{PCB}$, which is shown as an example in Figure 10, is fabricated as optimized using the GA-FEM approach.

For all the PCB fabricated coils, the system parameters are measured using a network analyzer reference VNA Master Anritsu MS2036A. This network analyzer was calibrated such that all cable influences are removed. The experimental system used to measure the efficiency of the fabricated coils is presented in Figure 11. This figure is showing particular arrangements made to ensure coil alignments with $30 \mathrm{~mm}$ separation distance between the primary and secondary coils.

For each design case, the network analyzer is used to perform two-port measurements from $1 \mathrm{MHz}$ to $20 \mathrm{MHz}$ on each pair of coils. To extract the experimental efficiency values, $S$-parameters are measured and then converted into $Z$-parameters using a dedicated MATLAB script $[33,34]$. The
TABLE 5: Analytical, numerical, and experimental results for all optimization approaches.

\begin{tabular}{lccc}
\hline Approaches & $\begin{array}{c}\text { Analytical } \\
\text { results }\end{array}$ & $\begin{array}{c}\text { Numerical } \\
\text { results }\end{array}$ & $\begin{array}{c}\text { Experimental } \\
\text { results }\end{array}$ \\
\hline According to [19] & $65.5 \%$ & $67.7 \%$ & $63.4 \%$ \\
Iterative approach & $78.6 \%$ & $70.4 \%$ & $68 \%$ \\
Genetic algorithms & $80.6 \%$ & $73.5 \%$ & $72 \%$ \\
GA-FEM-optimizer & & $78 \%$ & $77.9 \%$ \\
\hline
\end{tabular}

quality factor of each coil is determined using the following equations:

$$
\begin{aligned}
Q_{1} & =\frac{\operatorname{Im}\left(Z_{11}\right)}{\operatorname{Re}\left(Z_{11}\right)} ; \\
Q_{2} & =\frac{\operatorname{Im}\left(Z_{22}\right)}{\operatorname{Re}\left(Z_{22}\right)} .
\end{aligned}
$$

The coupling factor between the primary and secondary coil is expressed as

$$
k_{12}=\sqrt{\frac{\operatorname{Im}\left(Z_{12}\right) \operatorname{Im}\left(Z_{21}\right)}{\operatorname{Im}\left(Z_{22}\right) \operatorname{Im}\left(Z_{11}\right)}}
$$

where $Q_{1}, Q_{2}$, and $k_{12}$ are substituted in (8) to get the efficiency values. The obtained efficiency values for the different design cases are shown in Figure 12.

Referring to Figure 12, the efficiency values at $13.56 \mathrm{MHz}$ operating frequency are extracted and summarized in Table 5 together with the analytic and simulated results for the different approaches. This table clearly shows that the experimental results are in close agreement with what has been analytically and numerically predicted. Slight differences are observed between measured and simulated efficiency results. Comparing the different methods, the accuracy of GA-FEM is clear with only very small difference $(0.1 \%$ at $13.56 \mathrm{MHz})$ between measurements and simulations. This high accuracy can be explained by the fact that purely numerical optimization is implemented taking into account real resistances, parasitic capacitances, and all kinds of coupling effects between the coil and its environment.

Apart from GA-FEM accuracy, it is important to compare and validate the accuracy of the proposed IP and GA analytical approaches. Comparing analytical to experimental results from Table 5, 16\% errors are obtained for IP and only $12 \%$ errors are shown for GA. These are reasonable errors for both IP and GA if we consider the simplified mathematical models we used to approximate the coils electric parameters. These analytical models are derived with ignoring all types of losses such as capacitive, proximity effect and eddy current losses. Such losses make the actual coil resistances larger than those analytically predicted. Larger real resistance values generate lower coil quality factors and thus lower measured efficiencies are obtained in comparison with analytical values.

Comparing GA to IP, GA is more accurate; this is due to higher accurate inductance values predicted for coils optimized with GA. Actually, it is demonstrated according 


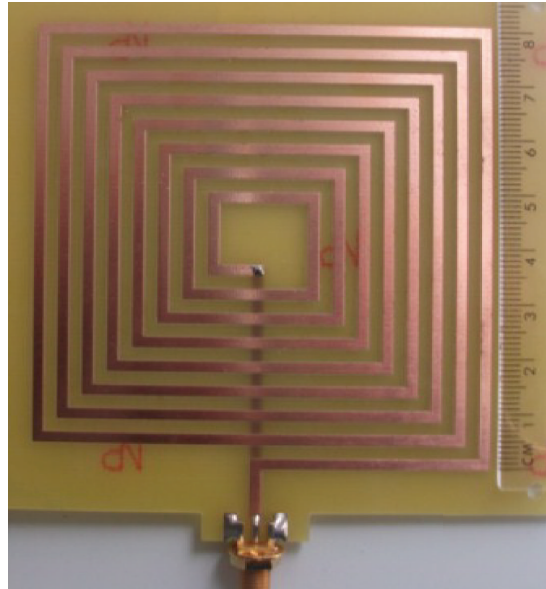

(a)

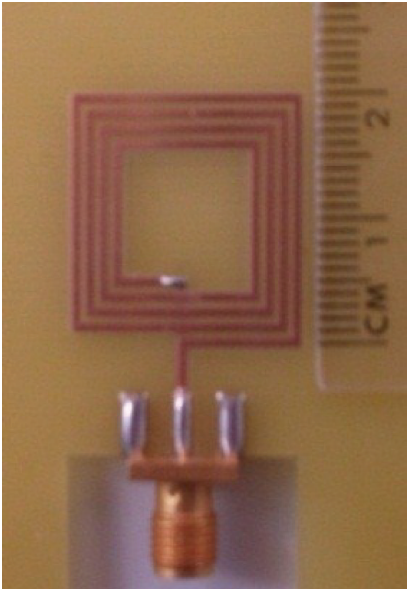

(b)

FIGURE 10: Photograph of the fabricated printed spiral coil optimized using GA-FEM: (a) primary coil and (b) secondary coil.

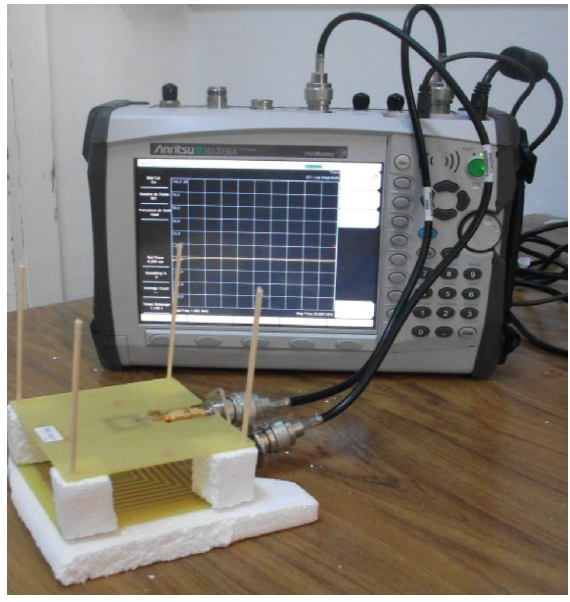

FIGURE 11: Experimental setup for measuring link efficiency using a network analyzer.

to [35] that the accuracy of the inductance model defined by (1) decreases for increasing $(s / w)$ ratios. Thus, if we compare the coil geometries optimized with IP and GA, it is clear from Tables 2 and 3 that $s_{1} / w_{1}=3$ and $s_{2} / w_{2}=2$ for the IP design versus $s_{1} / w_{1}=2$ and $s_{2} / w_{2}=1.34$ for GA. Thus, inductance values derived from (1) for both primary and secondary coils are more accurate for GA as studied in [35]. Actually, with GA, we were able to optimize both s- and w-parameters at the same time contrary to IP. This results in higher accuracy of GA in comparison with IP.

At the end, higher efficiency values are experimented for GA-FEM $(77.9 \%$ at $13.56 \mathrm{MHz})$ in comparison with GA $(72 \%$ at $13.56 \mathrm{MHz}$ ). Using GA-FEM as a reference design, GA is capable of delivering an accurate enough design with around $7 \%$ experimental performance degradation. This is acceptable given the approximate modeling used for GA. However, GAFEM requires a long simulation time to converge and one can use GA to initialize GA-FEM and thus considerably

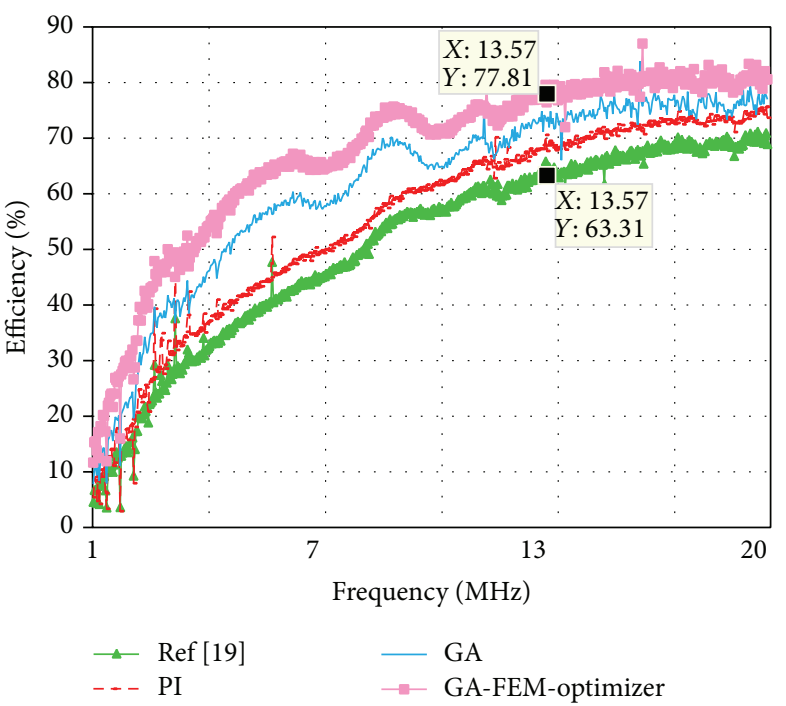

FIgUre 12: Measured efficiency values for GA-FEM, GA, and IP design optimizations compared to reference design [19].

accelerate its convergence speed with further performance improvements.

\section{Conclusions}

This paper aims for geometry optimization of inductively coupled printed spiral coils (PSC) for powering a given implantable sensor system. This is a complex nonlinear optimization problem with many interfering design parameters. Our objective is to implement and compare different optimization approaches capable of achieving, in a simple and a systematic way, optimal and accurate link designs within acceptable design times.

In this context, analytic approaches are first proposed. These approaches are based on simple mathematical models that approximate the electric parameters of the coils and the 
power transfer efficiency. Using these models, an Iterative Procedure (IP) is first developed. IP is a traditional popular approach for optimizing coil geometries. Such method suffers from ambiguity, heaviness, and complexity shortcomings. However, improved performance results are obtained using the proposed IP approach in comparison with a reference design for the same system. Advanced population based search strategies implementing Genetic Algorithms (GA) are then used. Actually, it was shown that GA is easier to implement and is capable of generating better optimized designs in a more systematic and efficient way. GA is also capable of delivering the adequate design within short computation times.

Both IP and GA analytical approaches are compared to GA-FEM, a purely simulation based optimization method. GA-FEM consists in coupling finite element simulations with GA based search. Performed simulation results confirmed that GA-FEM achieves, at the resonant frequency, the highest performance. In comparison with GA-FEM, acceptable 7\% efficiency losses are observed for GA. Despite its highest performance, GA-FEM suffers from extensive processing and very long design time. To minimize its processing time and further improve its performance, GA-FEM can be initialized using GA. In this case, even better performance results are obtained with higher convergence speed.

Experimental validation has been performed for all the designed cases. For this aim, the different coils optimized with the different approaches are fabricated on printed circuit boards (PCB). The system parameters are then measured and the transfer power efficiencies are determined. It is shown that the proposed IP and GA analytical approaches are accurate enough despite the approximated mathematical models used. Comparing GA to IP, GA is more accurate as we were able to better control particular design parameters. This leads to higher accurate inductances predicted for coils optimized with GA. Finally, the highest performance and accuracy of GA-FEM is confirmed with only minor errors observed between measurements and simulation results.

\section{Competing Interests}

The authors declare that they have no competing interests.

\section{Acknowledgments}

This project was funded by the King Abdulaziz City for Science and Technology (KACST), under Grant no. 91أت-34. The research team acknowledges and expresses their gratitude to KACST for the technical and financial support.

\section{References}

[1] A. Trigui, S. Mehri, A. C. Ammari, J. Ben Hadj Slama, and M. Sawan, "Prosthetic power supplies," in Wiley Encyclopedia of Electrical and Electronics Engineering, John Wiley \& Sons, New York, NY, USA, 2015.

[2] T. Sun, X. Xie, and Z. Wang, Wirless Power Management, Springer, New York, NY, USA, 2013.
[3] K. Van Schuylenbergh and R. Puers, The Concept of Inductive Powring, Springer Science+Business Media BV, Dordrecht, Netherlands, 2009.

[4] G. A. Kendir, W. Liu, G. Wang et al., "An optimal design methodology for inductive power link with class-E amplifier," IEEE Transactions on Circuits and Systems I: Regular Papers, vol. 52, no. 5, pp. 857-866, 2005.

[5] B. Lenaerts and R. Puers, "Inductive link design," in Omnidirectional Inductive Powering for Biomedical Implants, Analog Circuits and Signal Processing, pp. 39-81, Springer, Dordrecht, The Netherlands, 2009.

[6] A. Trigui, S. Hached, F. Mounaim, A. C. Ammari, and M. Sawan, "Inductive power transfer system with self-calibrated primary resonant frequency," IEEE Transactions on Power Electronics, vol. 30, no. 11, pp. 6078-6087, 2015.

[7] M. Kiani and M. Ghovanloo, "A figure-of-merit for designing high-performance inductive power transmission links," IEEE Transactions on Industrial Electronics, vol. 60, no. 11, pp. 52925305, 2013.

[8] U.-M. Jow and M. Ghovanloo, "Design and optimization of printed spiral coils for efficient transcutaneous inductive power transmission," IEEE Transactions on Biomedical Circuits and Systems, vol. 1, no. 3, pp. 193-202, 2007.

[9] H. Peng, J. Zhao, H. Zhang et al., "A novel approach to design of microstrip UWB bandpass filter using modified genetic algorithm," Progress In Electromagnetics Research M, vol. 36, pp. 169-175, 2014.

[10] J. T. Rayno, J. S. K. Nakatsu, G. C. Huang, N. Celik, and M. F. Iskander, "3D metamaterial broadband ground plane designed using genetic programming for the long slot array antenna," in Proceedings of the IEEE Antennas and Propagation Society International Symposium (APSURSI '13), pp. 400-401, Orlando, Fla, USA, July 2013.

[11] R. Bera, D. Mandal, R. Kar, and S. P. Ghoshal, "Application of improved Particle Swarm Optimization technique for thinning of concentric hexagonal array antenna," in Proceedings of the 4th World Congress on IEEE Information and Communication Technologies (WICT '14), pp. 188-193, Bandar Hilir, Malaysia, December 2014.

[12] M. Angelova and T. Pencheva, "Tuning genetic algorithm parameters to improve convergence time," International Journal of Chemical Engineering, vol. 2011, Article ID 646917, 7 pages, 2011.

[13] P. P. Chandran and S. Viswasom, "Gain and bandwidth optimization of a novel microstrip patch antenna," in Proceedings of the 4th International Conference on Advances in Computing and Communications (ICACC '14), pp. 315-318, IEEE, Cochin, India, August 2014.

[14] M. Ural and C. Bayseferogullari, "Solution of the antenna placement problem by means of global optimization techniques," in Proceedings of the 20th International Conference on Microwaves, Radar and Wireless Communications (MIKON '14), pp. 1-4, Gdańsk, Poland, June 2014.

[15] A. Pichler, D. Steffelbauer, and A. Nazarov, "Examples for genetic algorithm based optimal RFID tag antenna design," in Proceedings of the IEEE RFID Technology and Applications Conference (RFID-TA '14), pp. 223-227, IEEE, Tampere, Finland, September 2014.

[16] S. Song and R. D. Murch, "An efficient approach for optimizing frequency reconfigurable pixel antennas using genetic algorithms," IEEE Transactions on Antennas and Propagation, vol. 62, no. 2, pp. 609-620, 2014. 
[17] S. Mehri, J. Ben Hadj Slama, A. C. Ammari, and H. Rmili, "Genetic algorithm based geometry optimization of inductively coupled printed spiral coils for remote powering of electronic implantable devices," in Proceedings of the Global Summit on Computer and Information Technology (GSCIT '14), pp. 1-6, Sousse, Tunisia, June 2014.

[18] HFSS Online Help, April 2015, http://www.ansys.com/.

[19] E. G. Kilinc, C. Dehollain, and F. Maloberti, "Design and optimization of inductive power transmission for implantable sensor system," in Proceedings of the 11th International Workshop on Symbolic and Numerical Methods, Modeling and Applications to Circuit Design (SM2ACD '10), pp. 1-5, IEEE, Gammath, Tunisia, October 2010.

[20] A. K. RamRakhyani, S. Mirabbasi, and M. Chiao, "Design and optimization of resonance-based efficient wireless power delivery systems for biomedical implants," IEEE Transactions on Biomedical Circuits and Systems, vol. 5, no. 1, pp. 48-63, 2011.

[21] U.-M. Jow and M. Ghovanloo, "Modeling and optimization of printed spiral coils in air, saline, and muscle tissue environments," IEEE Transactions on Biomedical Circuits and Systems, vol. 3, no. 5, pp. 339-347, 2009.

[22] M. Zargham and P. G. Gulak, "Maximum achievable efficiency in near-field coupled power-transfer systems," IEEE Transactions on Biomedical Circuits and Systems, vol. 6, no. 3, pp. 228245,2012

[23] W. B. Kuhn and N. M. Ibrahim, "Analysis of current crowding effects in multiturn spiral inductors," IEEE Transactions on Microwave Theory and Techniques, vol. 49, no. 1, pp. 31-38, 2001.

[24] H. Kondori, M. A. Mansouri-Birjandi, and S. Tavakoli, "Reducing mutual coupling in microstrip array antenna using metamaterial spiral resonator," International Journal of Computer Science Issues, vol. 9, no. 3(1), pp. 52-56, 2012.

[25] L. Zhang, H. Chang, and R. Xu, "Equal-width partitioning roulette wheel selection in genetic algorithm," in Proceedings of the Conference on Technologies and Applications of Artificial Intelligence (TAAI '12), pp. 62-67, IEEE, Tainan, Taiwan, November 2012.

[26] A. Sharma and M. Sinha, "Influence of crossover and mutation on the behavior of Genetic algorithms in mobile Ad-hoc networks," in Proceedings of the 8th International Conference on Computing for Sustainable Global Development (INDIACom '14), pp. 895-899, New Delhi, India, March 2014.

[27] C. Pan, S. Cao, K. Zheng, and Z. He, "Optimizing appropriate two probable value method by adaptive genetic algorithm," in Proceedings of the International Conference on Computational Problem-Solving (ICCP '12), pp. 171-173, Leshan, China, October 2012.

[28] T. D. Nguyen, T. P. Vuong, Y. Duroc, and V. Y. Vu, "Optimization of pifa antenna using an auto-embedded genetic algorithm," in Proceedings of the $3 r$ International Conference on Communications and Electronics (ICCE '10), pp. 367-372, IEEE, Nha Trang, Vietnam, August 2010.

[29] Y. He, J. Ao, X. Tang, and J. Yang, "The optimum design of PIFA based on HFSS and genetic algorithm," in Proceedings of the 7th International Conference on Wireless Communications, Networking and Mobile Computing (WiCOM '11), pp. 1-4, Wuhan, China, September 2011.

[30] W.-W. Lee, S.-Y. Chen, T.-C. Lin, H.-T. Chou, and H.-T. Hsu, "Integration of HFSS and genetic algorithm for the optimum design of waveguide components," in Proceedings of the IEEE Antennas and Propagation Society International Symposium
(AP-S '07), pp. 2225-2228, IEEE, Honolulu, Hawaii, USA, June 2007.

[31] S. Shu-Hui and W. Bing-Zhong, "Parameter optimization based on GA and HFSS," Journal of Electronic Science and Technology of China, vol. 3, no. 1, pp. 45-47, 2005.

[32] A. J. Kerkhoff and H. Ling, "Design of a band-notched planar monopole antenna using genetic algorithm optimization," IEEE Transactions on Antennas and Propagation, vol. 55, no. 3, pp. 604-610, 2007.

[33] D. Pozar, Microwave Engineering, John Wiley \& Sons, New York, NY, USA, 2nd edition, 1998.

[34] M. Kiani, U.-M. Jow, and M. Ghovanloo, "Design and optimization of a 3-coil inductive link for efficient wireless power transmission," IEEE Transactions on Biomedical Circuits and Systems, vol. 5, no. 6, pp. 579-591, 2011.

[35] N. Troedsson and H. Sjöland, "Monolithic inductor modeling and optimization," in Radio Design in Nanometer Technologies, pp. 217-240, Springer, Berlin, Germany, 2006. 


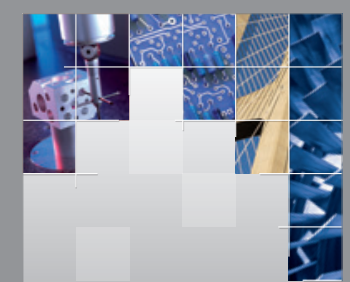

\section{Enfincering}
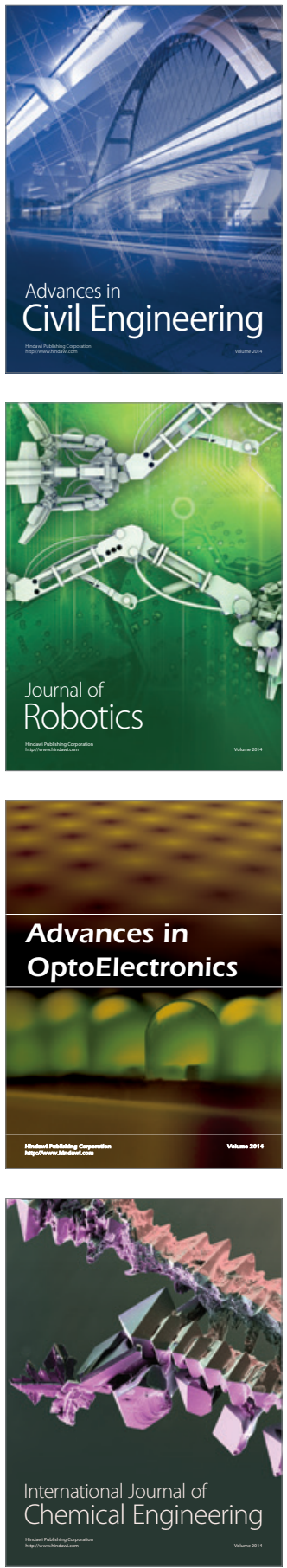

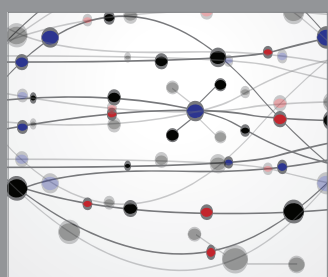

The Scientific World Journal

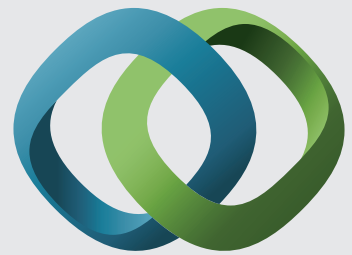

\section{Hindawi}

Submit your manuscripts at

http://www.hindawi.com
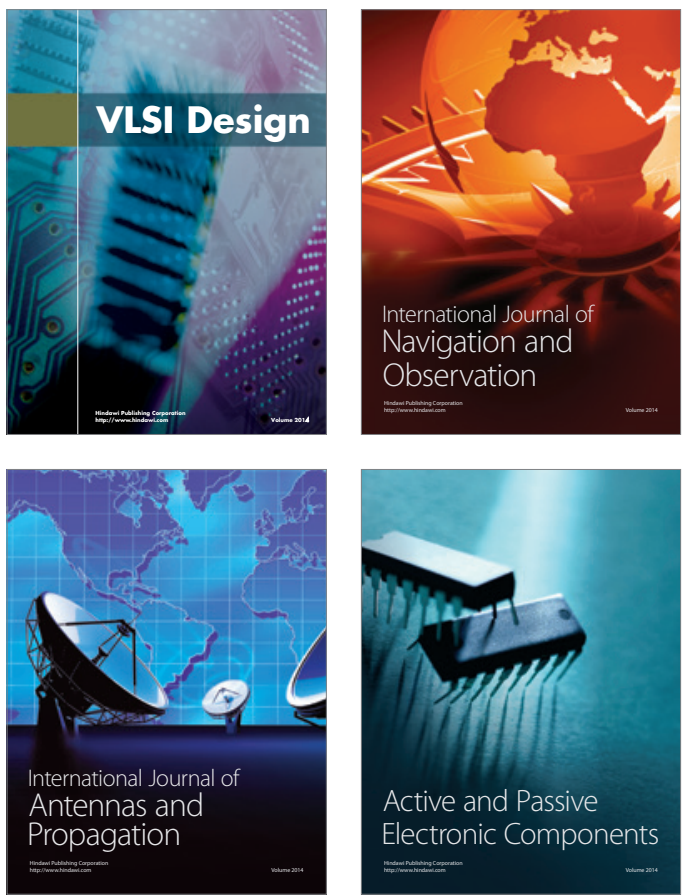
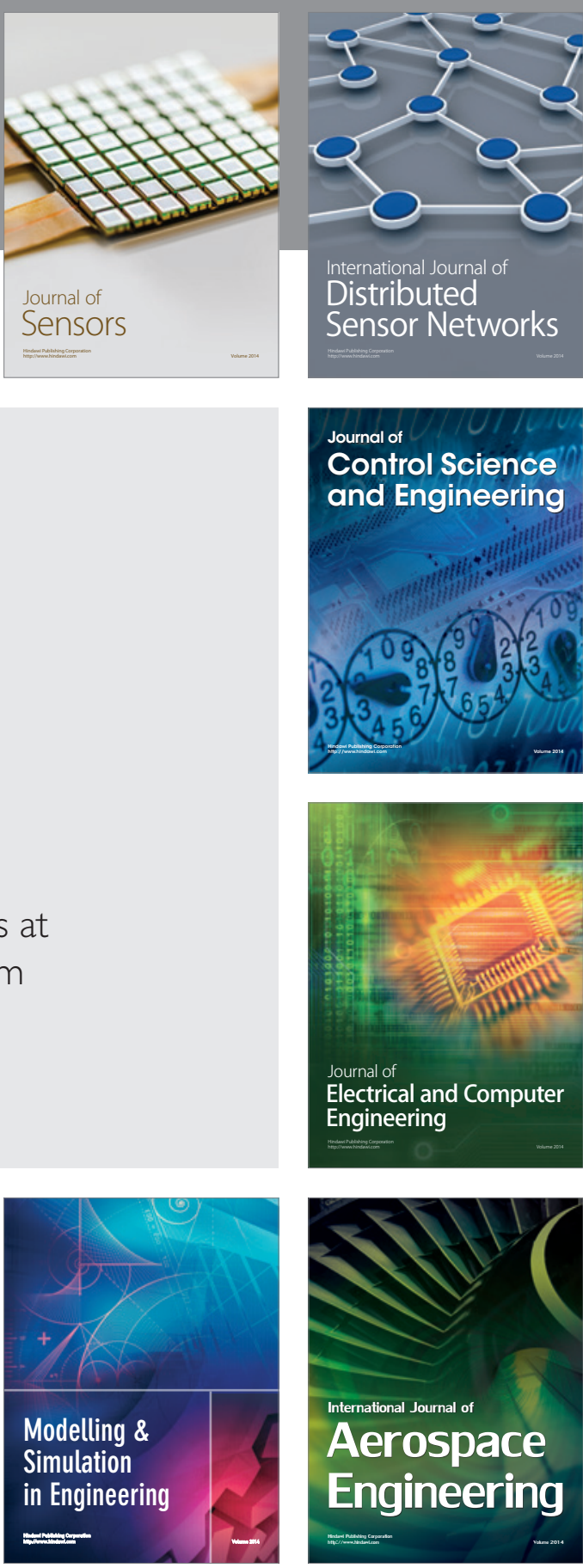

International Journal of

Distributed

Sensor Networks

Journal of

Control Science

and Engineering
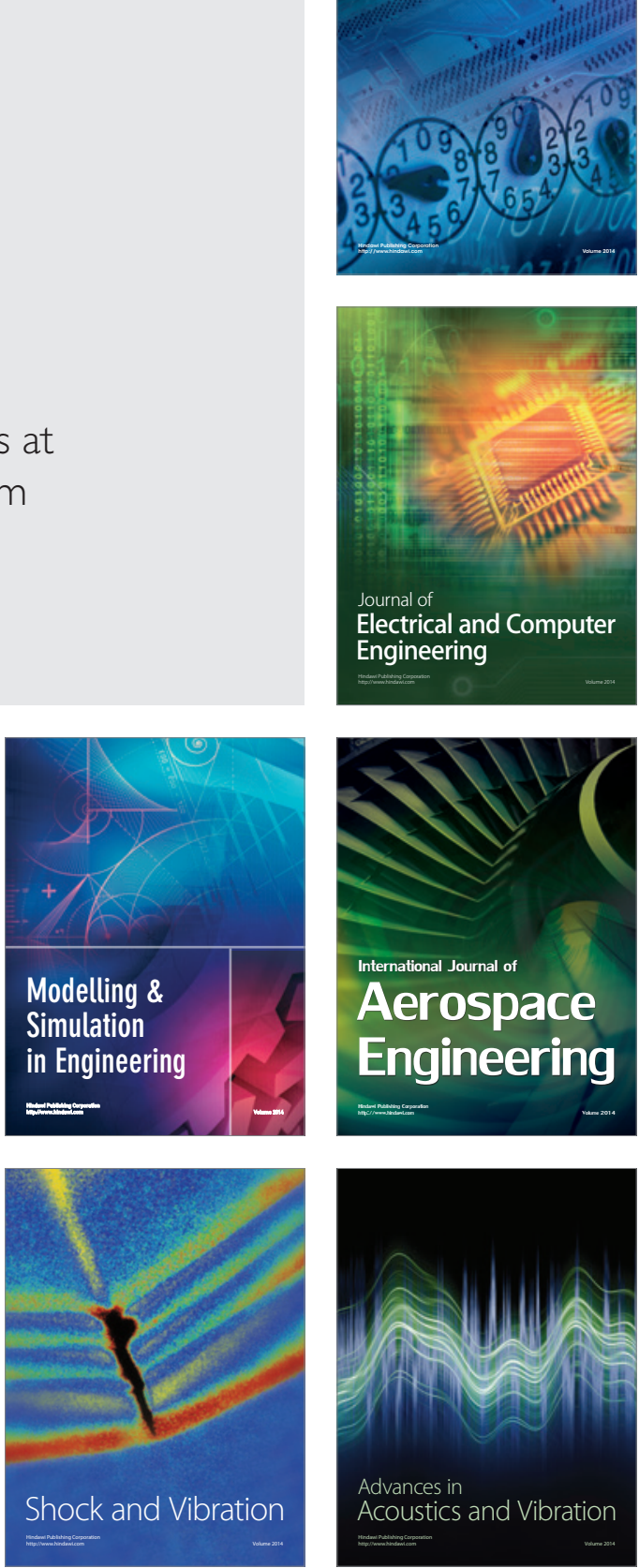